\title{
Analyses physicochimiques de différents thés commerciaux et risque de l'excès de fluor chez la population au Maroc
}

\author{
Issam ESSEBBAHI ${ }^{1}$, Chadia OUAZZANI ${ }^{1 *}$, Abdallah MOUSTAGHFIR ${ }^{2}$, \\ Abdallah DAMI ${ }^{1}$ et Lhoussine BALOUCH ${ }^{1}$ \\ ${ }^{1}$ Laboratoire de Biochimie et de Biologie Moléculaire, Faculté de Médecine et de Pharmacie, \\ Mohammed V, University of Rabat, Morocco. \\ ${ }^{2}$ Laboratoty of Research Odontological, Biomaterials and Nanotechnology, Faculty of Dentistry, \\ Mohammed V, University of Rabat, Morocco. \\ *Auteur correspondant ; E-mail : ouazcom@yahoo.fr
}

\section{RESUME}

Sous la forme de fluorure, le fluor entre dans la constitution des tissus durs comme les dents, les os et les cartilages. L'apport excessif en fluor pendant plusieurs mois ou plusieurs années lors de la période de formation des dents peut provoquer la fluorose. Certains aliments habituellement consommés au Maroc sont riches en fluor. Les traitements industriels de transformation, préparation et conservation mettent à la disposition du consommateur des aliments de coût moins chers et de compositions variables qui peuvent être nuisibles à la santé buccale. L'étude des qualités physicochimiques de différentes sources alimentaires de fluor constituées de différents thés noirs, verts, d'eaux de puits consommés par la population marocaine permet de dévoiler la corrélation entre la consommation des aliments riches en fluor et l'apparition de la fluorose dentaire. Différentes de tisanes de granules de 12 thés verts et noirs ont été préparés selon la variation de la durée d'infusion et du mode d'ébullition. Les teneurs de fluor, calcium, chlorures ont été déterminées par les méthodes spectrophotométriques sur 17 échantillons de thés verts et noirs commercialisés, thés glacés, thés de distributeur automatique, thé de buvette, thé de préparation familiale. La teneur en fluor a été déterminée sur 3 échantillons des eaux de puits, 3 eaux d'assainissement urbain de la région de Skhirat Témara afin de déterminer l'apport de fluor contenu dans l'eau de préparation des tisanes. Les analyses physicochimiques révèlent des taux de calcium de 14 à $60 \mathrm{mg} / \mathrm{l}$, chlorures $(14$ à $75 \mathrm{mg} / \mathrm{l})$ et de fluor $(0,93$ à $2,7 \mathrm{mg} / \mathrm{l})$ chez les différents thés verts et noirs étudiés, thé de distributeur automatique, thé de buvette et familiale. Les analyses des taux de fluor de 3 échantillons de thés verts marquent Sultan, Menara, Dahmis et un thé noir Earl Grey révèlent des valeurs supérieures aux normes recommandées pour les enfants et les adultes. Le taux de fluor du thé de la buvette est supérieur à celui du thé de distributeur automatique et de préparation familiale. Ces résultats permettent de prévenir la population face au risque d'atteinte de la fluorose dentaire, en limitant la quantité de fluor ingérée par les aliments consommés. Les analyses des niveaux de fluor de tous les types de produits de thés et des eaux de préparation des thés permettront de disposer de mesures de protection de consommation excessive de fluor.

(C) 2020 International Formulae Group. All rights reserved.

Mots clés : Fluor, aliments, thés, Maroc, excès, risques, fluorose. 


\title{
Physicochemical analyzes of different commercial teas and risk of excess fluorine in the population in Morocco
}

\begin{abstract}
In the form of fluoride, fluorine enters into the constitution of hard tissues such as teeth, bones and cartilage. Excessive intake of fluoride for several months or years during the period of tooth formation can cause fluorosis. Some foods usually consumed in Morocco are rich in fluorine. The industrial treatments of preparation, transformations make available to the consumer cheaper foods of variable composition which can be harmful to oral health. The study of physicochemical qualities of different food sources of fluorine consisting of different black teas, green teas and well water consumed by the population reveals a correlation between the consumption of foods rich in fluorine and the appearance of dental fluorosis. Different preparations of herbal teas from granules of 12 green and black teas were prepared according the variation of the infusion time, the boiling mode. The contents of fluorine, calcium, chlorides were determined by spectrophotometric methods on 17 samples of green and black teas from vending machines, refreshment tea, family preparation tea. The fluorine content was determined on 3 samples of well water, 3 urban sanitation water from the Skhirat Temara region in order to determine the fluorine content contained in the water for preparing herbal teas. The physicochemical analyzes reveal levels of Calcium (14 to $60 \mathrm{mg} / \mathrm{l})$, Chlorides (14 to $75 \mathrm{mg} / \mathrm{l})$ and fluorine $(0.93$ to $2.7 \mathrm{mg} / \mathrm{l})$ in the various green and black teas studied, tea vending machine, refreshment and family tea. The analyzes of the fluoride levels of three samples of marketed green teas Sultan, Menara, Dahmis and an Earl Gray black tea reveal values higher than the recommended standards for children's and adults. The fluoride level of the tea in the refreshment room is higher than that of the vending machine and family preparation tea. These results make it possible to warn the population facing the risk of developing dental fluorosis, by limiting the amount of fluorine ingested by the consumed food. Analyzes of fluoride levels of all types of tea products and tea preparation waters will provide measures to protect against excessive fluoride consumption.
\end{abstract}

(C) 2020 International Formulae Group. All rights reserved.

Keywords: Fluorine, foods, teas, Morocco, excess, risk, fluorosis.

\section{INTRODUCTION}

Le fluor est un élément essentiel pour le corps humain pour une teneur comprise entre 1,0 et 1,5 mg par jour (WHO, 2011). À faible dose, le fluor est important pour la croissance et pour le maintien du tissu osseux et des dents en prévenant la carie dentaire. Il constitue aussi un risque considérable pour la santé si la teneur dépasse $1,5 \mathrm{mg} /$ jour (WHO, 2019).

Plusieurs travaux ont mis en évidence différents mécanismes par lesquels le fluor perturbe la formation de l'émail et entraîne la formation d'un émail poreux inesthétique. Une ingestion excessive de fluorures entraîne l'inhibition des améloblastes, le changement des caractéristiques d'absorption et les propriétés de surface des cristaux d'émail (Tanimoto et al., 2008; Bronckers et al., 2009; Chandna et al., 2016) avec la formation d'un émail poreux inesthétique.

Les fluorures se retrouvent dans l'environnement à la fois naturellement (à travers l'érosion des roches et les émissions volcaniques) et à la suite de certaines activités humaines comme l'exploitation minière et le traitement du phosphate naturel, la fabrication d'aluminium ou encore la fluoration de l'eau de boisson (Kaseva, 2006 ; Janardhana Raju, 2017; Anirban et al., 2019). Certains aliments contiennent naturellement du fluor, c'est le cas par exemple du sel de table, du thé, des poissons, des épinards ou encore de certaines eaux minérales. Certains aliments comme le thé seraient une source d'exposition importante aux fluorures (Alenka et al., 2012). Les modes de conditionnement et de préparation des tisanes de thé différents selon les pays avec des conséquences sur la 
composition chimique pourraient être néfastes sur la santé (Astill et al., 2001).

Différents travaux, à travers le monde, ont révélé l'atteinte de la population par la fluorose dentaire (WHO, 2006 ; Goodarzi et al., 2016). Au Maroc, différents travaux ont révélé l'exposition de la population par les eaux de nappes souterraines des régions phosphatées exposant à la fluorose dentaire (Mountadar et al., 2010; El Jaouadi et al., 2014). Peu d'études ont été menées au niveau des sources alimentaires sur la toxicité liée aux fluorures chez la population marocaine. Au Maroc, la population se caractérise par son régime alimentaire très particulier marqué par une consommation élevée de thé dans lequel le fluorure est présent avec une teneur remarquable.

Cette étude vise à mettre en évidence les teneurs en fluor contenues dans des thés verts et thés noirs et dans les eaux de puits consommés par la population marocaine.

\section{MATERIEL ET METHODES Matériel}

Les qualités organoleptiques des différents échantillons de thés verts et thés noirs sont présentées dans le Tableau 1.

Les échantillons de thé bouillis sont récoltés respectivement dans un distributeur automatique, de la cafeteria d'un établissement public et d'une préparation familiale de thé vert à domicile. Les eaux de puits ont été prélevées durant la période de mars à avril 2019 dans la région rurale de Rabat Témara Skhirat dans des flacons stériles en polyéthylène et conservés à $4{ }^{\circ} \mathrm{C}$.

\section{Préparation des tisanes de thé}

Deux répétitions de 2 grammes de granules de chaque variété de thés sont pesées et sont additionnées à $40 \mathrm{ml}$ d'eau du robinet bouillant dans des béchers en pyrex et laissées infuser à température ambiante séparément pendant 10 min et $30 \mathrm{~min}$. Après filtration des résidus de feuilles, les extraits des tisanes sont récupérés et conservés en plusieurs aliquotes pour une durée ne dépassant pas $24 \mathrm{~h}$.

\section{Préparation des thés bouillis}

$2 \mathrm{~g}$ de chaque variété de thé sont additionnés à $120 \mathrm{ml}$ d'eau du robinet et mis à ébullition dans des théières domestiques pendant $5 \mathrm{~min}$. Les résidus de feuilles de thé sont éliminés par filtration et les extraits de thés sont conservés à $4{ }^{\circ} \mathrm{C}$ en plusieurs aliquotes pour une durée ne dépassant pas $24 \mathrm{~h}$.

\section{Dosage du calcium, des chlorures et du fluor}

Les taux de fluorures dans les tisanes de thé et dans les extraits de thés bouillis (en essais doubles) sont déterminés par la méthode colorimétrique de Belcher- West à l'Alizarine complexone (Tsunoda et al, 1985) dans des tubes en polyéthylène. En présence d'Alizarine complexone, le nitrate de lanthane donne une coloration rouge en milieu aqueux, en présence des anions $\mathrm{F}^{-}$, il se forme un complexe de coloration bleue. Le nouveau complexe est soluble dans l'acétone, et dosé par spectrophotométrie à $618 \mathrm{~nm}$. L'absorbance des échantillons est retranchée à celle d'un témoin dans lequel l'extrait de thé est remplacé par l'eau distillée. La concentration du fluor des échantillons est calculée par analyse par rapport à la courbe d'étalonnage.

Les taux de calcium sont déterminés par la méthode CPC (Moorehead et al, 1974). Cette méthode est basée sur le réactif o-cresol phtalein complexon en milieu alcalin $(1 \mathrm{ml})$ réagit avec le calcium des solutions d'extraits de thés pour former un complexe qui absorbe à $570 \mathrm{~nm}$ après incubation pendant $5 \mathrm{~min}$ à la température ambiante. L'absorbance des échantillons est retranchée à celle d'un témoin dans lequel l'extraits de thé est remplacé par l'eau distillée. La concentration des taux de calcium des échantillons est déterminée par référence à un étalon de calcium. 
Les taux de chlorure sont déterminés par la méthode colorimétrique (Florence et al., 1971 ; Tietz et al., 1999). Cette méthode est basée sur la réaction du thiocyanate mecurique non dissocié pour former du chlorure mercurique non dissocié et des ions thiocyanates libres. Les ions thiocyanates réagissent avec le fer ferrique pour former un complexe coloré en rouge dont l'absorbance est déterminée à 400-500 $\mathrm{nm}$. La concentration des taux de chlorures des échantillons est déterminée par référence à un étalon de chlorures.

Tableaux 1 : Caractéristiques organoleptiques des différents échantillons de thés étudiés.

\begin{tabular}{|c|c|c|c|}
\hline $\begin{array}{l}\text { Échantillons } \\
\text { Composition }\end{array}$ & $\begin{array}{l}\text { Nature } \\
\text { Couleur }\end{array}$ & $\begin{array}{l}\text { Nom commercial } \\
\text { Emballage }\end{array}$ & Origine \\
\hline $\mathrm{F} \% / \mathrm{G} \% / \mathrm{B} \%$ & Thé vert & Sultan/ & Maroc \\
\hline $195-05$ & Marron vf & Papier all fermé & \\
\hline \multirow[t]{2}{*}{$290-10$} & Thé vert & Violan & Maroc \\
\hline & Vert gris & Papier all fermé & \\
\hline \multirow[t]{2}{*}{$320 / 40 / 40$} & Thé vert & Caravane (extra) & Maroc \\
\hline & Vert $\mathrm{m}$ & Papier ouvert & \\
\hline \multirow{2}{*}{$4 \quad 70 / 20 / 10$} & Thé vert & Al massrar & Légère \\
\hline & Vert $\mathrm{m} \mathrm{cl}$ & Papier all fermé & \\
\hline \multirow[t]{2}{*}{$5 \quad 70 /-/ 30$} & Thé vert & La Menara & Maroc \\
\hline & Vert bou & Papier ouvert & \\
\hline \multirow[t]{2}{*}{$6 \quad 95 /-/ 5$} & Thé vert & Essbaa (le lion) & Maroc \\
\hline & Vert gris & Papier all fermé & \\
\hline \multirow[t]{2}{*}{$7 \quad 75 /-/ 25$} & Thé vert & Caravane (Dahbia) & Maroc \\
\hline & Vert foncé & Papier ouvert & \\
\hline \multirow[t]{2}{*}{$8 \quad 95 /-/ 25$} & Thé vert & Dahmiss & Maroc \\
\hline & Vert gris & Papier ouvert & \\
\hline \multirow{2}{*}{$\begin{array}{l}\text { Très petits } \\
\text { granuleux H }\end{array}$} & Thé noir & Lipton & Anglo- \\
\hline & Marron $\mathrm{f}$ & Boîtes sachets & Néerlandais \\
\hline \multirow{2}{*}{$\begin{array}{l}10 \text { Poudre de } \\
\text { granules }\end{array}$} & Thé noir & Earl Grey & $\begin{array}{ll}\text { France } & \text { Faible }\end{array}$ \\
\hline & Marron $\mathrm{f}$ & Boite sachets & \\
\hline \multirow[t]{2}{*}{11 Batonnets } & Thé noir & Caylan & Maroc Légère \\
\hline & Marron f & Sachet fermé & \\
\hline \multirow{2}{*}{$\begin{array}{l}12 \text { Granules } \\
\text { très fins } H\end{array}$} & Thé noir & Sultan & Maroc \\
\hline & Marron f & Sachet fermé & \\
\hline \multirow[t]{2}{*}{13 Liquide } & Thé glacé & Casino & France fruité \\
\hline & & Bouteille & \\
\hline \multirow[t]{2}{*}{14 Liquide } & Thé glacé & Lipton & Anglo-néerlandais \\
\hline & & Bouteille & \\
\hline 15 Liquide & Inconnu & $\begin{array}{l}\text { Thé de distributeur } \\
\text { automatique }\end{array}$ & Inconnu \\
\hline 16 Liquide & Thé vert & Thé de buvette & Inconnu \\
\hline 17 Liquide & Thé vert & Thé de maison & Inconnu \\
\hline
\end{tabular}




\section{RESULTATS}

\section{Variation du pH, taux de calcium, taux de chlorures}

L'analyse des constituants minéraux calcium et chlorures des granules de thés verts et noirs présente un intérêt nutritionnel et permet de révéler la diversité des différents thés étudiés.

La mesure du $\mathrm{pH}$ permet de mesurer l'acidité ou l'alcalinité des différents thés étudiés. L'acidité est un facteur qui peut ramollir l'émail et exposer les dents au risque de la fluorose (Werguet, 2013).

Les résultats de la variation du $\mathrm{pH}$ du taux de calcium, de chlorures, fluor sont cités dans le Tableau 2. Les analyses physicochimiques des thés glacés, thé de buvette et thé de préparation familiale étudiés révèlent des valeurs de $\mathrm{pH}$ comprises entre 4,30 et 7,03 . Deux thés glacés et le thé de distributeur automatique ont un $\mathrm{pH}$ acide. L'analyse de calcium révèle la présence des taux de calcium variables entre 14 à $60 \mathrm{mg} / \mathrm{l}$. Les taux des chlorures varient entre 14 et $75 \mathrm{mg} / \mathrm{l}$. La teneur en calcium dosée dans les échantillons d'extraits de thés correspond à la composition de calcium contenue dans les feuilles de thés additionnée à celle de l'eau utilisée pour la préparation des tisanes.

\section{Variation du taux de fluor des tisanes des thés verts}

Les concentrations du taux de fluor dans les thés verts varient selon les marques de thés, selon le temps d'infusion et selon le mode de préparation. La concentration du fluor pour les préparations des tisanes de thé de 10 minutes d'infusion varie entre $0,5 \mathrm{mg} / \mathrm{l}$ et $1,62 \mathrm{mg} / \mathrm{l}$ correspondant à un taux de 0,5 à $2,51 \mathrm{mg} / 100 \mathrm{~g}$ de poids frais du thé vert (Figure 1). Pour les préparations de 30 minutes d'infusion, le taux de fluor se situe entre 0,93 à $2,7 \mathrm{mg} / \mathrm{l}$ correspondant à 1,39 à $3,64 \mathrm{mg} / 100 \mathrm{~g}$ de poids frais de thé vert. Par la méthode d'ébullition, les taux de fluor varient de 1,04 à $1,77 \mathrm{mg} / 1$ soit 4,16 à 7,52 $\mathrm{mg} / 100 \mathrm{~g}$ de poids frais de thé vert (Figure 1). Les valeurs du taux de fluor des tisanes de thés de la préparation de $30 \mathrm{mn}$ des variétés Sultan, Menara et Dahmis sont plus élevées par rapport aux autres thés étudiés.

\section{Variation du taux de fluor des thés noirs}

Les concentrations du taux de fluor dans thés noirs sont comprises entre $0,48 \mathrm{mg} / \mathrm{l}$ et $1,26 \mathrm{mg} / \mathrm{l}$ pour les infusions de $10 \mathrm{~min}$ et entre $1,27 \mathrm{mg} / \mathrm{l}$ et $1,75 \mathrm{mg} / \mathrm{L}$ pour dans les infusions de 30 minutes (Figure 2). Le taux de fluor du thé noir marque Earl Grey est supérieur à celui des autres thés noirs étudiés.

Variation du taux de fluor des thés glacés, thé de distributeur automatique thé de buvette et de préparation familiale

Les analyses de la teneur en fluor révèlent des valeurs de $1,34 \mathrm{mg} / \mathrm{L}, 0,65 \mathrm{mg} / \mathrm{l}$, $1,02 \mathrm{mg} / \mathrm{L}, \quad 1,73 \quad \mathrm{mg} / \mathrm{l} \quad$ et $1,27 \mathrm{mg} / \mathrm{L}$ respectivement chez les thés glacés, thé de distributeur automatique, de buvette et de préparation familiale (Figure 3). Le taux de fluor du thé des buvettes est supérieur à celui des thés glacés, de distributeur et des préparations familiales. Le thé glacé d'origine France présente un taux de fluor supérieure à celui du thé glacé d'origine Anglonéerlandais.

\section{Teneur de fluor des eaux de préparation des tisanes et thés bouillis}

L'eau est un élément essentiel qui rentre dans la préparation des thés, présente un intérêt dans le processus d'infusion et des qualités organoleptiques des tisanes et des thés bouillis. L'analyse du taux de fluor des eaux permet de déterminer l'apport en fluor des différentes eaux de préparation, des échantillons des eaux de puits prélevées de la région Rabat Témara Skhirat. L'analyse des eaux des puits révèle des taux de fluor variant de $0,4 \mathrm{mg} / \mathrm{l}$ à $0,56 \mathrm{mg} / \mathrm{l}$ alors que le fluorure est quasi absent dans les eaux de robinets (Figure 4). 
Tableaux 2 : Variation des $\mathrm{pH}$, du taux de calcium et de chlorure des différentes tisanes de thés verts et noirs, thés glacés, thé de distributeur automatique, thé de buvette et de préparation familiale.

\begin{tabular}{|c|c|c|c|c|}
\hline & Échantillons & pH & $\mathrm{Ca}^{2+}(\mathrm{mg} / \mathrm{l})$ & $\mathrm{Cl}^{-}(\mathrm{mg} / \mathrm{l})$ \\
\hline $\mathrm{Tr}$ & & & 15 & 16 \\
\hline \multirow{8}{*}{ Thé vert } & 1 & 7,05 & 33 & 17 \\
\hline & 2 & 6,86 & 46 & 27 \\
\hline & 3 & 6,85 & 38 & 34 \\
\hline & 4 & 6,74 & 27 & 22 \\
\hline & 5 & 6,62 & 42 & 17 \\
\hline & 6 & 6,88 & 33 & 53 \\
\hline & 7 & 6,83 & 41 & 47 \\
\hline & 8 & 6,92 & 38 & 55 \\
\hline \multirow{4}{*}{ Thé noir } & 9 & 6,18 & 44 & 67 \\
\hline & 10 & 6,05 & 42 & 75 \\
\hline & 11 & 6,18 & 60 & 72 \\
\hline & 12 & 6,16 & 14 & 60 \\
\hline \multirow{2}{*}{ Thés glacé } & 13 & 4,62 & 59 & 28 \\
\hline & 14 & 4,30 & 23 & 59 \\
\hline $\begin{array}{c}\text { Thé de distributeur } \\
\text { automatique }\end{array}$ & 15 & 4,43 & 48 & 64 \\
\hline Thé de buvette & 16 & 6,77 & 30 & 52 \\
\hline $\begin{array}{c}\text { Thé de préparation } \\
\text { familiale }\end{array}$ & 17 & 6,81 & 36 & 46 \\
\hline
\end{tabular}

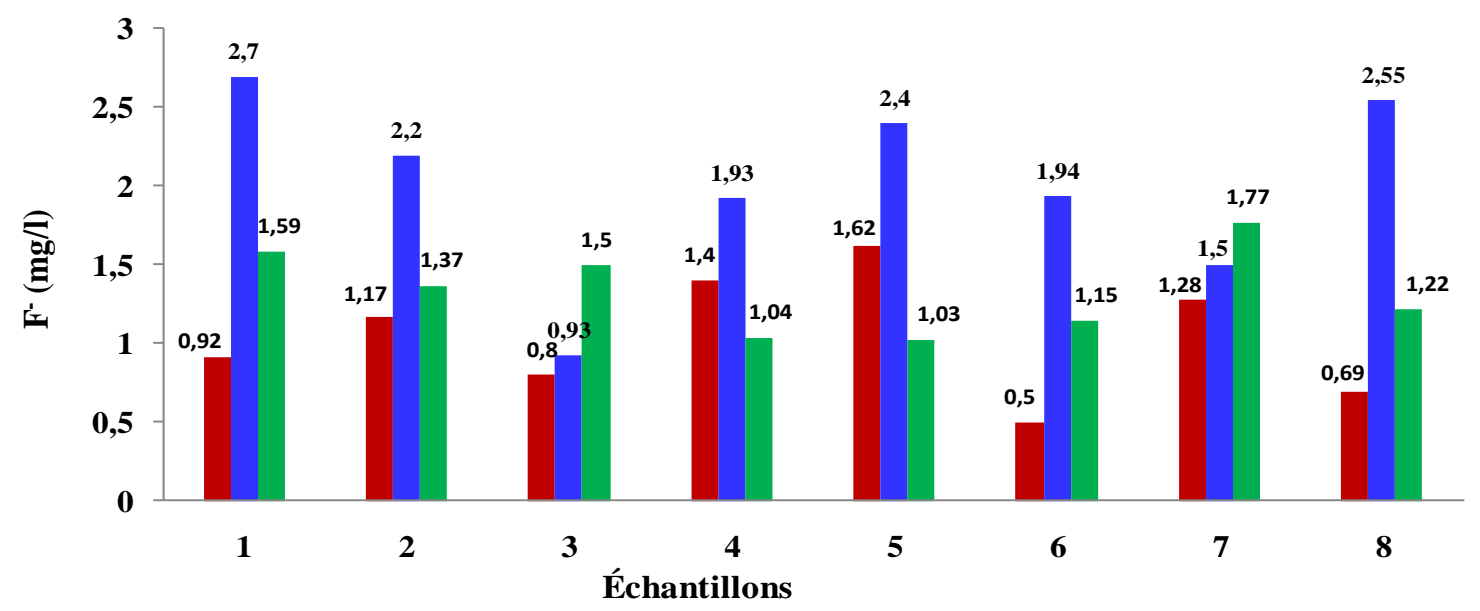

Temps d'infusion $\square \mathbf{1 0}$ minutes $\square 30$ minutes $\square$ Préparation traditionnelle

(Préparation traditionnelle : familiale)

Figure 1 : Taux de fluor en mg/l dans les thés verts. 


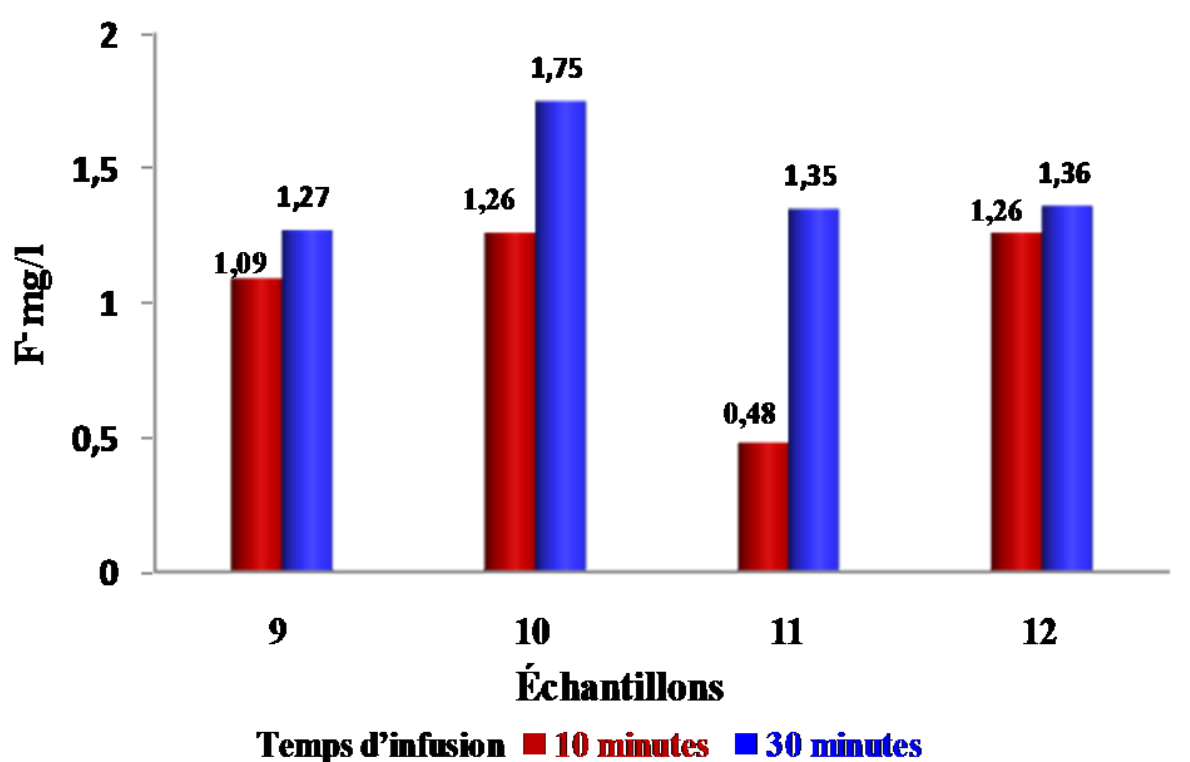

Temps d'infusion des thés 10 et 30 minutes

Figure 2: Taux de fluor dans les thés noirs (échantillons 9,10, 11 et 12).

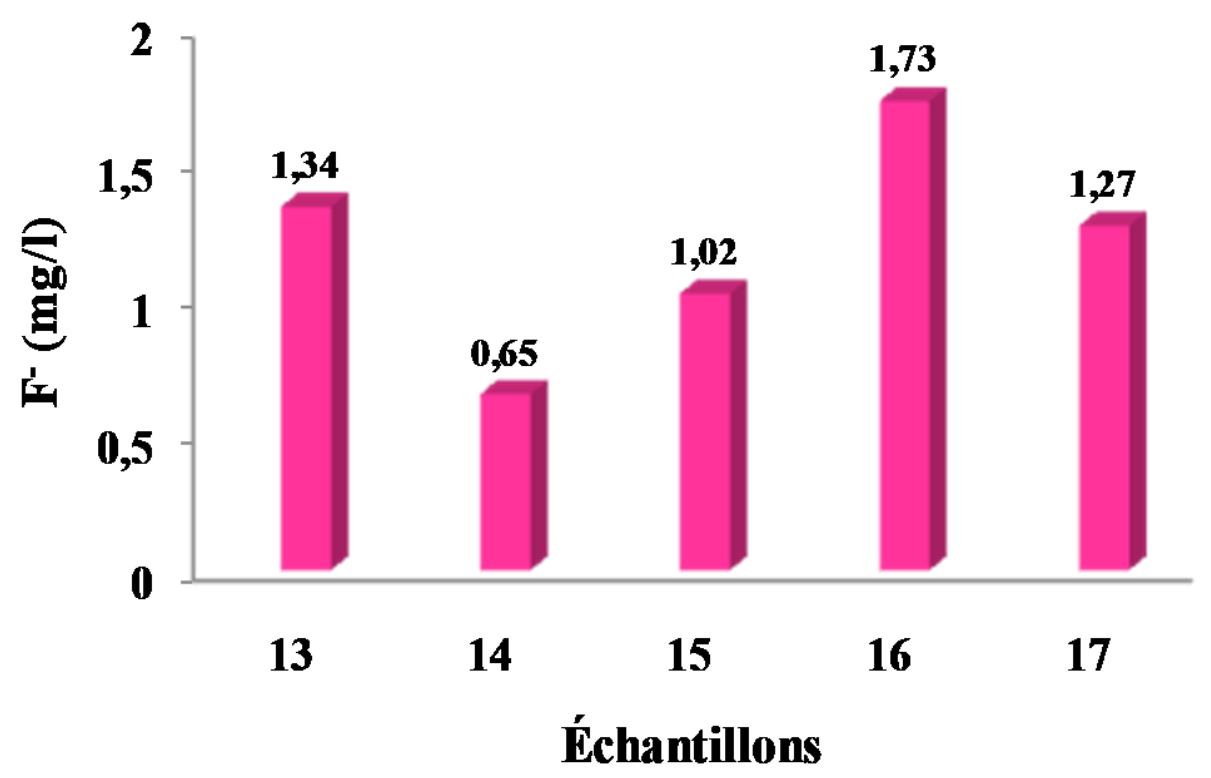

Figure 3 : Taux de fluor dans les thés glacé (échantillons 13 et 14), thé de distributeur automatique (échantillon 15), thé de buvette (échantillon 16) et the préparation familiale (échantillon 17). 


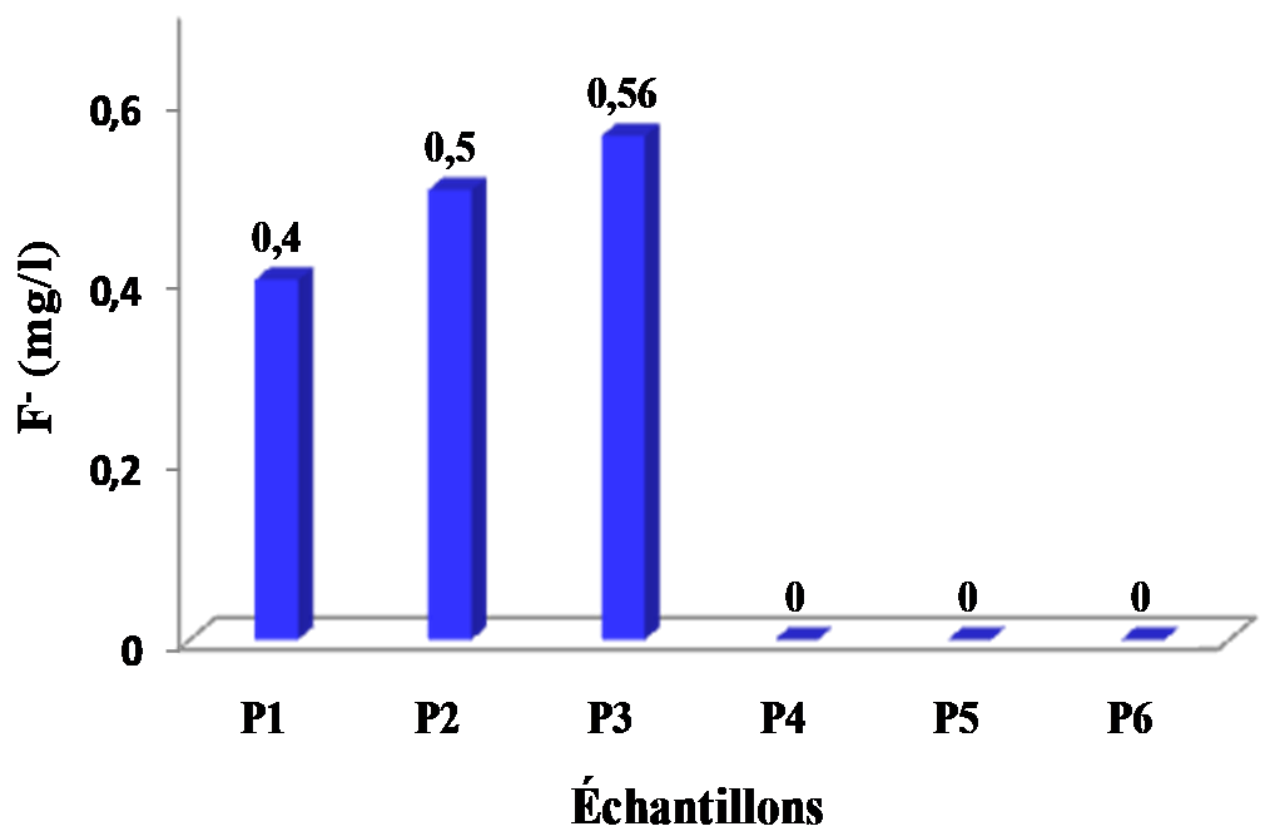

Figure 4 : Taux d'eau d fluor des eaux de puits (P1, P2, P3) et des eaux de robinets (P4, P5, P6).

\section{DISCUSSION}

Les analyses physico-chimiques des tisanes des thés verts, thés noirs, thés glacés, thé de de distributeur automatique, thé de buvette et thé de préparation familiale révèlent des valeurs de $\mathrm{pH}$ comprises entre 4,30 et 7,03. L'acidité élevée des thés glacés est due à la présence des composants acides tels que l'acide ascorbique et certaines saveurs. L'acidité du thé de distributeur automatique est due à la présence de citron et les émulsifiants. Cette acidité contenue dans ces thés présente un risque potentiel d'endommager les dents. Des études subséquentes ont démontré qu'une déminéralisation de l'émail de la dent est atteinte lorsque celle-ci est soumise à un environnement de $\mathrm{pH}$ inférieur au seuil de 5,5 (Werguet, 2013). Les taux de calcium des thés noirs et verts sont constitués dans l'intervalle de référence normales, $1000 \mathrm{mg} /$ jour pour les adultes (FAO/WHO, 2001).

Les teneurs en chlorures des thés noirs sont supérieures à celles des thés verts et sont constituées dans l'intervalle de référence, 250 $\mathrm{mg} / \mathrm{L}$ recommandé par l'OMS (WHO, 2003).
Les taux de fluor révélés durant les temps d'infusion de 30 min sont supérieurs à celles de $10 \mathrm{~min}$. Les teneurs en fluor après une infusion de $30 \mathrm{~min}$ atteignent des valeurs $2,7 \mathrm{mg} / \mathrm{l}$ (thés verts), $1,8 \mathrm{mg} / \mathrm{l}$ (thés noirs) et $1,73 \mathrm{mg} / \mathrm{l}$ pour le thé de consommation de buvette. Ces résultats sont semblables aux études précédentes citant des valeurs de 0,32 à 4,54 $\mathrm{mg}$ de fluorures par litre d'infusion de thés noirs (Malinowska et al., 2008) et 0,57 à $3,72 \mathrm{mg} / \mathrm{l}$ de thés noirs infusés pendant 5 minutes (Emekli et al., 2009). Les valeurs de fluor des thés de préparation de $30 \mathrm{mn}$ des variétés Sultan, Menara et Dahmis sont supérieures aux normes recommandées pour les enfants et les adultes (Martin, 2001). Les valeurs élevées du taux de fluor déterminées dans les granules de thé pourraient être corrélées avec les qualités organoleptiques (couleur, faible odeur feuilles séchées, conditionnement papier ouvert) chez les variétés de thé vert (Sultan, Menara et Dahmi) et thé noir Earl Grey. D'autres études ont révélé l'influence des conditions de culture, l'âge des feuilles sur la composition chimique 
des thés et des qualités organoleptiques (Krieps, 2009).

Dans notre étude, les eaux de consommation des puits de la région rurale est faible par rapport à d'autres régions minières ou industrielles ou le taux est plus élevé (Lebrahimi et al., 2017).

Des études récentes ont révélé la corrélation entre l'excès de fluor contenu dans les eaux consommées des eaux souterraines des régions volcaniques au Mexique chez une population d'enfants et l'atteinte de la fluorose dentaire (Irigoyen-Camacho et al., 2016). Dans notre étude, le fluor contenu dans les eaux de préparation des thés est une source supplémentaire à celle des thés, additionnée à celle des pâtes dentifrices (Prystupa, 2011), et d'autres aliments susceptibles d'être consommés les légumes, le poisson.

La teneur de fluor des thés et le mode de consommation de la population révèlent des risques de toxicité par le fluor et l'atteinte de la fluorose dentaire. Ce risque est d'autant plus élevé selon les habitudes alimentaires et les coutumes. Les résultats observés chez une préparation familiale sont susceptibles d'augmenter chez les grands consommateurs de thés avec des variations sur la quantité de granules de thé utilisés et la durée d'ébullition

\section{Conclusion}

Les teneurs en fluor sont variables selon la nature des thés consommés par la population, la quantité et la durée de consommation. Les teneurs du taux de fluor des thés bouillis sont supérieures à cellles des thés des tisanes d'infusion de $30 \mathrm{~min}$ et 10 min. 3 types de thés verts (Sultan, Menara et Dahmi) et 1 thé (Earl Grey) noir présentent des taux de fluor supérieurs aux normes recommandées. Les taux de fluor des eaux de préparation des thés prélevés dans les eaux de puits sont supérieurs à celui des eaux d'assainissement urbain.

Les habitudes alimentaires et la vie en milieu rural pourraient induire une surconsommation involontaire de fluorures et, par conséquent, une augmentation de la prévalence des fluoroses dentaires.
D'autres études sur les paramètres température de l'eau, rapport masse feuille/ eau durée d'infusion permettront de relever les habitudes alimentaires, le mode de vie prédisposant la population à l'atteinte de la fluorose dentaire.

Le contrôle de la qualité physico chimique des eaux de préparation des thés permettra de prévenir le consommateur face à un excès de consommation en fluor.

Des dosages de fluor salivaires sur les dents permettront de constituer des diagnostics de la fluorose dentaire nécessaire pour la prise en charge et les suivis des patients chez le praticien dentiste.

Un contrôle de consommation des aliments fluore doit être effectué chez les enfants (avant 6 ans) afin de limiter la consommation des aliments riches en fluor.

\section{CONFLIT D'INTERETS}

Les auteurs ont affirmé qu'il n'y avait aucun conflit d'intérêt.

\section{CONTRIBUTIONS DES AUTEURS}

IE a réalisé les analyses et a rédigé le premier format du manuscrit sous les avis et le suivi de $\mathrm{CO}$ et $\mathrm{AM}$. $\mathrm{CO}$ et $\mathrm{AM}$ ont supervisé le travail, ont fait la relecture et ont corrigé la version révisée. $\mathrm{AD}$ et $\mathrm{EB}$ ont soutenu ce travail pour l'amélioration de la qualité scientifique du manuscrit.

\section{REMERCIEMENTS}

Les auteurs remercient toutes les personnes qui ont contribué d'une manière ou d'une autre à la réalisation de cette étude.

\section{REFERENCES}

Alenka K, Gašper T, Maja PS. 2012. Fluoride in teas of different types and forms and the exposure of humans to fluoride with tea and diet. Food chemistry, 130 (2): 286-290.

Anirban C, Mrinal KA, Arnab M, Prasanta D, Debasis D. 2019. Critical review on geochemical and geological aspects of fluoride belts, fluorosis and natural materials and other sources for 
alternatives to fluoride exposure. Journal of Hydrology, 574: 333-359.

Astill C, Birch MR, Dacombe C, Humphrey PG, Martin PT. 2001. Factors Affecting the Caffeine and Polyphenol Contents of Black and Green Tea Infusions. J Agric. Food Chem., 49: 5340-5347.

Bronckers ALJJ, Lyaruu DM, DenBesten PK. 2009. The Impact of Fluoride on Ameloblasts and the Mechanisms of Enamel Fluorosis. J Dent Res, Oct; 88(10): 877-893.

Chandna P, Srivastava N, Ali S. 2016. Remineralizing agents: The Next Frontier. Curr. Clin. Pharmacol., 11(3): 211-220.

DOI: 10.2174/1574884711666160607084813

El Jaouadi R, Ait El Cadi M, Bouslimane Y, Fekhaoui M, Bouklouze A, Cherrah Y. 2014.Teneur en fluorures des eaux de puits des régions rurales au Maroc. Tropical Dental Journal., 37(146): 4248.

Emekli AE, Yarat A, Akyuz S. 2009. Fluoride levels in various black tea, herbal and fruit infusions consumed in Turkey. Food and Chemical Toxicology, 47(7): 1495-1498.

DOI: 10.1016/j.fct.2009.03.036.

FAO/WHO. 2001. Human Vitamin and Mineral Requirements. Report of a joint FAO/WHO expert consultation Bangkok, Thailand. file:///C:/Users/HP/Desktop/FAO\%20\%2 0OMS\%20\%20\%20\%202001.pdf

Florence TM, Farrar YJ. 1971. Spectrophotometric determination of chloride at parts-per-billion level by mercury (II) thiocyanate method. Anal. Chim. Acta, 54: 373-377.

Goodarzi F, Mahvi AH, Hosseini M, Nedjat S, Nabizadeh Nodehi R, Kharazifard MJ, Parvizishad M, Cheraghi Z. 2016. The prevalence of dental fluorosis and exposure to fluoride in drinking water: A systematic review. J. Dent. Res. Dent Clin. Dent Prospects. 10(3): 127-35. DOI: 10.15171/joddd.2016.021.

Irigoyen-Camacho ME, García PA, Mejía GA, Huizar AR. 2016. Nutritional status and dental fluorosis among schoolchildren in communities with different drinking water fluoride concentrations in a central region in Mexico. Science of The Total Environment, 54115: 512-519

Janardhana Raju N. 2017. Prevalence of fluorosis in the fluoride enriched groundwater in semi-arid parts of eastern India: Geochemistry and health implications Quaternary International, 443 (Part B10) July.

Kaseva ME. 2006. Contribution of trona (magadi) into excessive fluorosis a case study in Majiya Chai ward, northern Tanzania. Sci. Total. Environ., 366 (1): 92-100.

DOI:

10.1016/j.scitotenv.2005.08.049

Krieps M. 2009. Thèse le the : origine, actualité et potentialités université Henri Poincaré - Nancy 1 faculté de pharmacie http://docnum.univ-

lorraine.fr/public/scdpha_t_2009_krieps_ marthe.pdf

Lebrahimi H, Fekhaoui M, Bellaouchou MA, Elabidi M, Tahri L. 2017. Determination of the fluoride Ions in the Groundwater in the Region of Khouribga (Morocco). Der Pharma Chemica, 9(8): 133-138.

Malinowska E, Inkielewicz I, Czarnowski W, Szefer P. 2008. Assessment of fluoride concentration and daily intake by human from tea and herbal infusions. Food Chem. Toxicology, 46(3): 1055-1061. DOI: 10.1016/j.fct.2007.10.039.

Martin A, AFSSA Apports nutritionnels conseillés pour la population française, 3e éd. (Retirage 2018) Janvier 2001. Reproduction Nutrition Development, EDP Sciences, 2001, 41 (2), pp.119-128. ff10.1051/rnd:2001100f .

Moorehead WR, Biggs HG. 1974. 2-Amino2-methyl-1-propanol as the Alkalizing Agent in an Improved Continuous-Flow Cresolphthalein Complexone Procedure for Calcium in Serum. Clinical Chemistry, 20(11): 1458-1460. DOI: 10.1093/clinchem/20.11.1458 
Mountadar M, Garmes H, Bouraji A, Yousrani K. 2010. Contamination des eaux souterraines et des sols par tes fluorures et leur impact à proximité des mines et d'usines d'exploitation des phosphates (Maroc). Journal Europeen d'Hydrologie, Cahiers de l'ASEES, 5(1): 61-69.

Prystupa J. 2011. Fluorine-A current literature review. An NRC and ATSDR based review of safety standards for exposure to fluorine and fluorides. Toxicol. Mech. Methods, 21(2): 103-170. DOI: $10.3109 / 15376516.2010 .542931$

Tanimoto K, Le T, Zhu L, Chen J, Featherstone JDB, Li W, DenBesten P. 2008. Effects of fluoride on the interactions between amelogenin and apatite crystals. J Dent Res., 87(1): 3944. DOI: $10.1177 / 154405910808700106$

Tietz NW. 1999. Text Bock of Clinical Chemistry $\left(3^{\text {rd }}\right.$ Edn), Burtis CA, Ashwood ER (eds). W.B. Saunder; 1063 - 1064, p. 1104.

Tsunoda H, Yu MH. 1985. Selected Papers from the $14^{\text {th }}$ Conference of the International Society for Fluoride Research. Fluoride Research, Japan. P312.
Werguet M. 2013. Les Erosions Dentaires, Données Actuelles. Thèse de Doctorat. Université de Lorraine Faculté De Chirurgie Dentaire, $\mathrm{N}^{\circ} 6082$.

World Health Organization (WHO). 2006. Fluoride Drinking Water, Fawell J, Bailey K, Chilton J, Dahi E, Fewtrell L, Magara Y (eds). Printed by TJ International (Ltd): Padstow, Cornwall, UK.

World Health Organization. 2003. Chloride in Drinking-water Background document for development WHO Guidelines for Drinking-water Quality. WHO reference number: WHO/SDE/WSH/03.04/03

World Health Organization. 2011. Guidelines for drinking-water quality (Fourth edition), Fluroride, chapter 12.1, pp. 371-373.

World Health Organization. 2019. Exposure to inadequate or excess fluoride: A major public health concern. https://apps.who.int/iris/bitstream/handle /10665/329484/WHO-CED-PHE-EPE19.4.5-eng.pdf?ua=1. 